\title{
A continuous sampling scheme for edge illumination $x$-ray phase contrast imaging
}

C. K. Hagen, ${ }^{1, a)}$ P. Coan, ${ }^{2,3}$ A. Bravin, ${ }^{4}$ A. Olivo, ${ }^{5}$ and P. C. Diemoz ${ }^{5}$

1) Department of Medical Physics and Biomedical Engineering, University College London, London WC1E 6BT, United Kingdom.

2) Faculty of Physics, Ludwig Maximilians University, 81377 Munich, Germany

3) Department of Clinical Radiology, Ludwig Maximilians University, 81377 Munich, Germany

4) European Synchrotron Radiation Facility, 38043 Grenoble Cedex, France

5) Department of Medical Physics and Biomedical Engineering, University College London, Malet Place, London WC1E 6BT, United Kingdom.

(Dated: 10 July 2015)

We discuss an alternative acquisition scheme for edge illumination (EI) x-ray phase contrast imaging (XPCi) based on a continuous scan of the object, and compare its performance to that of a previously used scheme, which involved scanning the object in discrete steps rather than continuously. By simulating signals for both continuous and discrete methods under realistic experimental conditions, the effect of the spatial sampling rate is analysed with respect to metrics such as image contrast and accuracy of the retrieved phase shift. Experimental results confirm the theoretical predictions. Despite being limited to a specific example, the results indicate that continuous schemes present advantageous features compared to discrete ones. Not only can they be used to speed up the acquisition, but they also prove superior in terms of accurate phase retrieval. The theory and experimental results provided in this study will guide the design of future EI experiments through the implementation of optimized acquisition schemes and sampling rates.

PACS numbers: 87.59.-e

Keywords: X-ray Imaging, Phase Contrast, Synchrotron Radiation, Sampling

a)Electronic mail: charlotte.hagen.10@ucl.ac.uk 


\section{INTRODUCTION}

In x-ray phase contrast imaging (XPCi), contrast is driven by the phase shift that x-rays suffer while they travel through matter. Local variations in this phase shift translate into small deviations of x-rays from their path (x-ray refraction). This can offer a promising alternative to conventional, attenuation-based x-ray imaging for the visualization of weakly attenuating specimens, due to the fact that phase effects are often stronger in this case ${ }^{1}$. To date, several XPCi modalities have been developed ${ }^{2-7}$. The edge illumination (EI) method stands out due to its high phase sensitivity and versatility ${ }^{8-10}$. In particular, the method was shown to be applicable both with highly coherent synchrotron radiation, as well as with incoherent radiation from conventional x-ray tubes ${ }^{11}$. The method is naturally suited to the latter since it is non-interferometric and achromatic ${ }^{12}$. The working principle of EI is shown in Fig. 1: a slit collimates the beam upstream of the object, typically down to 10-20 $\mu \mathrm{m}$, and a second slit, positioned in front of the detector, stops half of the beam, while the remaining half is allowed through. This "edge illumination" configuration creates sensitivity to refraction in addition to attenuation: when x-rays are refracted towards the uncovered detector area, an increased intensity is measured. Vice versa, when x-rays are refracted towards the covered area (downwards in Fig. 1(a)), a decreased intensity is measured. By repositioning the slit such that the other edge is illuminated ("opposing EI configuration" [Fig. 1(b)]), the effect of the refraction direction on the measured intensity is reversed. An image can be obtained by scanning the object vertically through the setup, a requirement that is eliminated if both slits are replaced by aperture masks which split the beam into an array of "beamlets" and repeat the EI configuration over the entire field of view of an area detector ${ }^{11}$. The latter is equivalent to the slit setup, provided the beamlets remain physically separated.

Without any processing, images show a combination of attenuation and refraction contrast, the latter having a "differential" nature and thus being strongest at sharp transitions within the object ${ }^{13}$. In fact, the refraction angle is expressed by $\alpha=(\lambda / 2 \pi) \cdot \partial \Phi / \partial y$, where $\Phi$ is the phase shift and $\lambda$ is the wavelength. Separate attenuation and refraction maps can be extracted through a procedure based on the acquisition of two images in opposing EI configurations ${ }^{8,14}$. From refraction angle images, the phase shift can be recovered via one-dimensional integration ("phase retrieval"), as required for truly quantitative imaging 

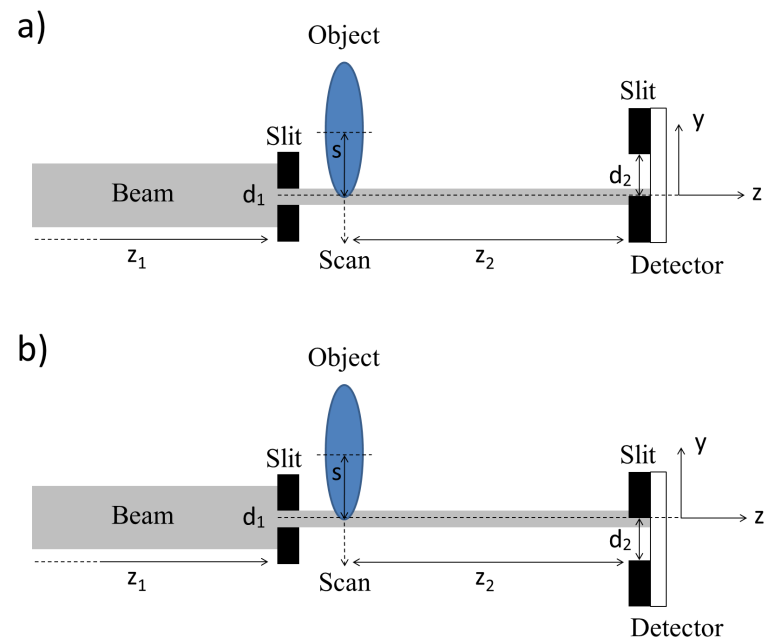

FIG. 1. Working principle of EI. Panels a) and b) show the setup in opposing edge illumination configurations.

and computed tomography $(\mathrm{CT})^{15}$.

The spatial sampling rate in EI is determined by the step size of the scan, or by the period of the sample mask in the full-field implementation of EI if additional scanning is not performed ${ }^{16,17}$. According to Nyquist's theorem ${ }^{18,19}$, aliasing is avoided if a signal is sampled at or above twice its highest spatial frequency (Nyquist frequency, $f_{N}$ ). The Nyquist frequency is determined by the object itself and the resolution limit of the system. Since EI is a differential XPC technique, signals can contain high spatial frequencies, especially at the edges of object features due to the measurement of a derivative. Consequently, optimal sampling requires high sampling rates, implying high dose delivery and long scan times. Therefore, a previous study investigated the effect of sampling rates below $f_{N}{ }^{17}$. It demonstrated that planar images of the phase shift can be severely affected, to a degree that the quantitative accuracy is lost.

In that previous study, a discrete sampling scheme was used, meaning that the object was kept in a fixed position while the detector was acquiring, and then stepped before the acquisition of the next frame ("discrete acquisition"). Here, we propose the use of an alternative approach in which the object is moved continuously by a certain distance (step size) while the detector is acquiring and kept in a fixed position during detector read-out ("continuous acquisition"). We use a theoretical approach to simulate and compare continuous and discrete EI acquisitions of a wire phantom under realistic experimental conditions. 
We analyze how the sampling rate affects a) the contrast in unprocessed (i.e. not phaseretrieved) images and b) the quantitative accuracy of the phase shift obtained through phase retrieval. Experimental results obtained using synchrotron radiation are presented, which support the theory. The presented formulas and general data analysis strategy will guide future EI acquisitions, both in terms of which acquisition scheme to use and how to select the sampling rate.

\section{THEORY}

Let us assume an experimental setup as shown in Fig. 1 and a parallel beam of unit intensity. The $z$-axis is assumed to intersect the $y$-axis at $y=0$. The effect of an object in the beam is described by its complex transmission function $q(y ; \lambda)=\sqrt{T(y ; \lambda)} \cdot \exp \{i \Phi(y ; \lambda)\}$, where:

$$
\begin{aligned}
& T(y ; \lambda)=\exp \left\{-\frac{4 \pi}{\lambda} \int \beta(y, z ; \lambda) d z\right\} \\
& \Phi(y ; \lambda)=\frac{2 \pi}{\lambda} \int \delta(y, z ; \lambda) d z
\end{aligned}
$$

are the beam transmission and phase shift, respectively. The parameters $\beta$ and $\delta$, which form the complex refractive index $(n=1-\delta+i \beta)$, denote the attenuation and phase shifting properties of the object. The transmission function of the first slit can be written as the following box function:

$$
\Pi_{d_{1}}(y)= \begin{cases}0, & \text { if }|y|>\frac{d_{1}}{2} \\ 1, & \text { if }|y| \leq \frac{d_{1}}{2}\end{cases}
$$

The intensity distribution on the detector plane for the object scan position $s$ can then be written $\mathrm{as}^{20}$ :

$$
I(y, s ; \lambda)=\left|\left[q(y-s ; \lambda) \cdot \Pi_{d_{1}}(y)\right] \star h_{z_{2}}(y ; \lambda)\right|^{2} \star g(y),
$$

where $h_{z_{2}}$ is the Fresnel propagator evaluated at distance $z_{2}, g(y)$ is the source distribution projected onto the detector plane and $\star$ indicates convolution. Assuming an ideal detector response, the recorded signal is given by the intensity distribution integrated over the uncovered detector area:

$$
S_{\text {ideal }}(s ; \lambda)=\int_{0}^{d_{2} / 2} I(y, s ; \lambda) d y
$$


Likewise, in the opposing edge illumination condition this is given by:

$$
S_{\text {ideal }}^{\text {opp }}(s ; \lambda)=\int_{-d_{2} / 2}^{0} I(y, s ; \lambda) d y .
$$

Equations 5 and 6 represent ideal EI signals obtained with an infinitely high sampling rate. In a real experiment, however, finite sampling needs to be used due to time and dose

restrictions. When the object is scanned in a discrete manner with step size $\Delta s$, data at the $n$th scanning step are given by $S_{\text {discr }}\left(\Delta s, s_{0}, n ; \lambda\right)=S_{\text {ideal }}\left(s_{0}+(n-1) \Delta s ; \lambda\right)$, (or by the corresponding expression for the opposing EI configuration). The parameter $s_{0}$ denotes the object position at the start of the scan; together with the step size $\Delta s$, this determines the exact sampling locations. When the proposed continuous acquisition scheme is employed instead, the signal is given by the following integral:

$$
S_{\text {cont }}\left(\Delta s, s_{0}, n ; \lambda\right)=\frac{1}{\Delta s} \int_{-\Delta s / 2}^{\Delta s / 2} S_{\text {ideal }}\left(s_{0}+(n-1) \Delta s-s ; \lambda\right) d s,
$$

which can also be expressed in form of a convolution:

$$
S_{\text {cont }}\left(\Delta s, s_{0}, n ; \lambda\right)=\frac{1}{\Delta s}\left[S_{\text {ideal }} \star \Pi_{\Delta s}\right]\left(s_{0}+(n-1) \Delta s ; \lambda\right) .
$$

Equations 5 to 8 allow simulating EI signals acquired with discrete and continuous schemes for different experimental parameters. In particular, the spatial sampling rate $\left(f_{s}\right)$ can be varied since it is related to the step size via $f_{s}=1 / \Delta s$.

\section{SIMULATIONS}

Data were simulated for a polyethylene terephthalate (PET) wire of $500 \mu \mathrm{m}$ in diameter. Parameters $z_{1}, z_{2}, d_{1}$ and $d_{2}$ were chosen to match a specific experimental setup implemented at beamline ID17 of the European Synchrotron Radiation Facility (ESRF), described below. Moreover, a Gaussian shaped source distribution with a full width half maximum (FWHM) dimension of $24 \mu \mathrm{m}$ and a $47 \mathrm{keV}$ beam energy were assumed. Theoretical $\beta$ - and $\delta$-values for PET at $47 \mathrm{keV}$ were obtained from an online database ${ }^{21}$. To first approximation, the spatial resolution limit in EI is given by the minimum between $d_{1}$ and $\max \left(\sigma, \sqrt{z_{2} \lambda}\right)$, where $\sigma$ denotes the dimension of the source projected onto the detector plane ${ }^{16}$. For the simulated experimental conditions, $d_{1}=20 \mu \mathrm{m}, \sigma \approx 0.6 \mu \mathrm{m}$ and $\sqrt{z_{2} \lambda} \approx 9.5 \mu \mathrm{m}$, thus the resolution limit is approximately $9.5 \mu \mathrm{m}$. Simulations were run for step sizes $(\Delta s)$ ranging from 50 
$\mu \mathrm{m}$ to $0.1 \mu \mathrm{m}$, corresponding to sampling rates $\left(f_{s}\right)$ ranging from 0.02 samples $/ \mu \mathrm{m}$ to 10 samples $/ \mu \mathrm{m}$. Due to it being much smaller than the resolution limit, the smallest simulated step $(\Delta s=0.1 \mu \mathrm{m})$ ensures that no undersampling artefacts occur in the ideal signals [Eqs. $5,6]$. The parameter $s_{0}$ was varied from $-350 \mu \mathrm{m}$ to $-300 \mu \mathrm{m}$, such that the object was located outside the beam at the start of the scan. The $50 \mu \mathrm{m}$ variation for $s_{0}$ was selected to cover a distance between at least two adjacent sampling points for all step sizes; this ensures that all possible outcomes for different $s_{0}$ are detected.

Simulated signals were analysed as a function of the step size $(\Delta s)$ and the parameter $s_{0}$, and the following metrics were considered. First, the contrast in the unprocessed (non-phase retrieved) signals was calculated as the relative signal difference between the maximum value at the left edge of the wire and the background:

$$
C\left(\Delta s, s_{0}\right)=\frac{S_{\text {edge }}\left(\Delta s, s_{0}\right)-S_{\text {background }}}{S_{\text {background }}}
$$

The dependence on $\lambda$ has been dropped to shorten the expression. It should be noted that the use of the right edge of the wire would lead to equivalent results, since the ideal intensity profiles are almost perfectly symmetric [see Fig. 2]. Second, refraction angles were extracted using two signals simulated at opposite EI configurations ${ }^{8,14}$, from which phase shifts were retrieved via numerical integration:

$$
\Phi\left(\Delta s, s_{0}, n\right)=\Delta s \cdot \frac{2 \pi}{\lambda} \sum_{n^{\prime}=1}^{n} \alpha\left(\Delta s, s_{0}, n^{\prime}\right) .
$$

For evaluation of the phase shifts accuracy, its maximum value was extracted:

$$
\Phi_{\max }\left(\Delta s, s_{0}\right)=\max _{n} \Phi\left(\Delta s, s_{0}, n\right)
$$

For a cylindrical object like a wire this is located in the centre of the retrieved profile.

\section{EXPERIMENTAL VERIFICATION}

Experiments were performed at beamline ID17 of the ESRF. The source-to-object and propagation distances were approximately $140 \mathrm{~m}\left(z_{1}\right)$ and $3.45 \mathrm{~m}\left(z_{2}\right)$, respectively. The source has full width half maximum (FWHM) dimensions of about $123 \mu \mathrm{m}(\mathrm{H}) \times 24 \mu \mathrm{m}$ (V). The beam was monochromatized by a fixed-exit Laue/Laue silicon $(1,1,1)$ crystal to an energy of $47 \mathrm{keV}(\Delta E / E \approx 0.02 \%)$. The detector was the FReLoN CCD camera ${ }^{22}$ coupled 
to a $40 \mu \mathrm{m}$ thick $\mathrm{Gd}_{3} \mathrm{Ga}_{5} \mathrm{O}_{12}$ scintillator, resulting in an effective pixel size of $46 \mathrm{x} 46 \mu \mathrm{m}^{2}$. A slit placed upstream the object collimated the beam to $20 \mu \mathrm{m}$ vertically $\left(d_{1}\right)$. No horizontal collimation was applied. A second slit with a vertical opening of $250 \mu \mathrm{m}\left(d_{2}\right)$ was placed in front of the detector and shifted such that $50 \%$ of the collimated beam fell on the active detector area [see Fig. 1].

The imaged object was a PET fibre (Goodfellow, UK) with a nominal diameter of 500 $\mu \mathrm{m}$. The object was scanned in continuous and discrete modes with a step size of $20 \mu \mathrm{m}$ $(\Delta s)$, which corresponds to a sampling rate of 0.05 samples $/ \mu \mathrm{m}\left(f_{s}\right)$. The exposure time was $2 \mathrm{~s}$ per scanning step in both cases. In order to also obtain data for a lower sampling rate $\left(\Delta s=40 \mu \mathrm{m} ; f_{s}=0.025\right.$ samples $\left./ \mu \mathrm{m}\right)$, the images acquired with the discrete scheme were subsampled along the scanning direction by a factor of two, and a two-pixel binning was applied to the image acquired with the continuous scheme. Images were corrected for dark current and flat field variations, and contrast and retrieved phase shift were calculated according to Eqs. 9 and 11.

\section{RESULTS AND DISCUSSION}

\section{A. Effect on Contrast}

Figures 2(a) to (c) show simulated profile signals of a wire for continuous (red line, circles) and discrete (blue line, squares) acquisition schemes and different step sizes $(\Delta s=$ 50, 20,1 $1 \mathrm{~m})$, corresponding to sampling rates of $f_{s}=0.02,0.05$ and 1 samples $/ \mu \mathrm{m}$. In these examples, the wire was initially positioned at $s_{0}=-350 \mu \mathrm{m}$ in all cases. Additionally, each panel contains the respective ideal signal (black line). Note that the ideal signal was simulated according to Eq. 5 and that it therefore already contains any blurring effects due to the imaging system. The sharp fringes at the edges of the wire are a characteristic feature of differential XPCi techniques, and contrast is effectively determined by the peak height. For lower sampling rates $(\Delta s=50,20 \mu \mathrm{m})$, it can be observed that the contrast is reduced compared to the ideal case. On the other hand, for a high sampling rate $(\Delta s=1$ $\mu \mathrm{m})$, contrast is practically identical to the ideal case. The same behaviour is illustrated in more detail in Figs. 2(d) and (e), where the contrast extracted from all simulated signals $\left(\Delta s=50 \mu \mathrm{m}\right.$ to $0.1 \mu \mathrm{m}, s_{0}=-350 \mu \mathrm{m}$ to $\left.-300 \mu \mathrm{m}\right)$ is displayed as a function of $\Delta s$ and $s_{0}$. 
Generally, it can be seen that contrast increases with higher sampling rates.
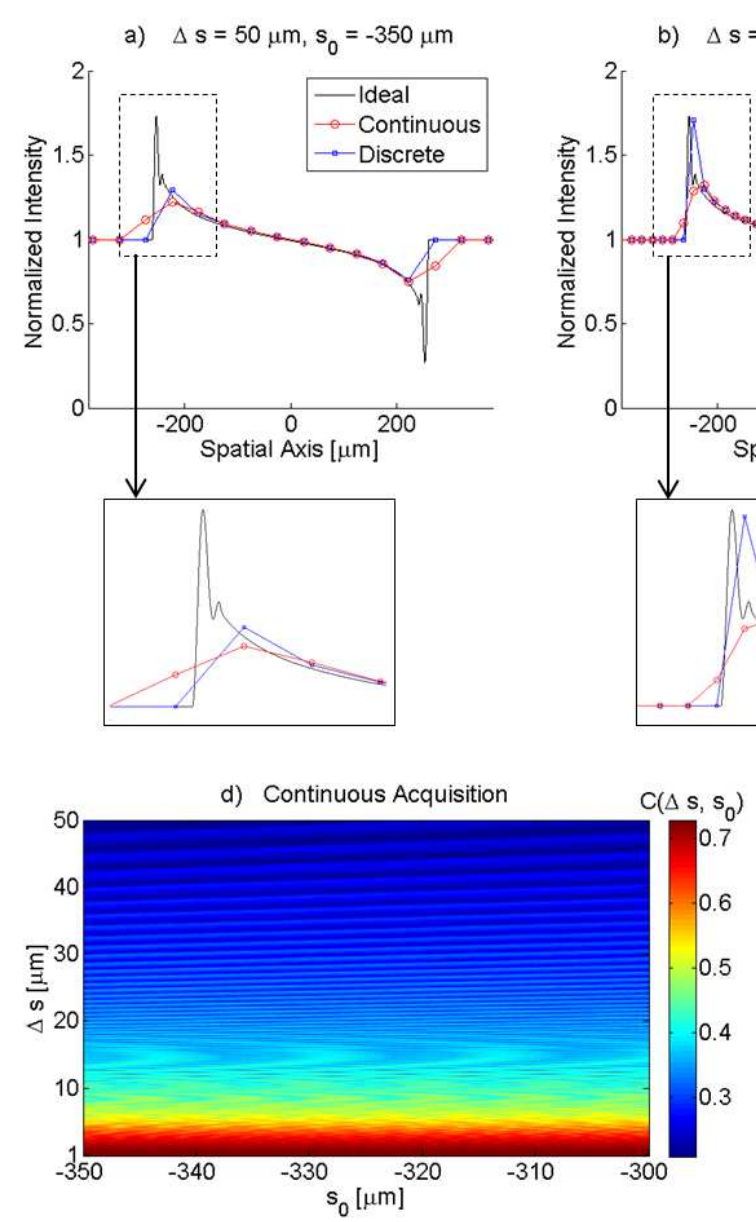

b) $\Delta \mathrm{s}=20 \mu \mathrm{m}, \mathrm{s}_{0}=-350 \mu \mathrm{m}$
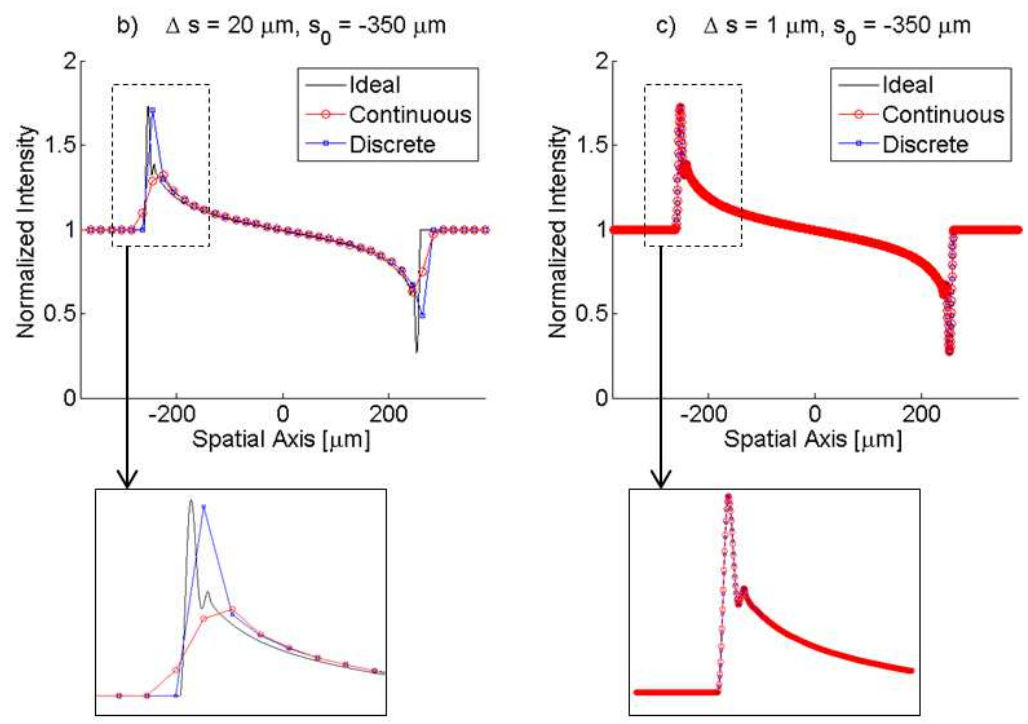

e) Discrete Acquisition

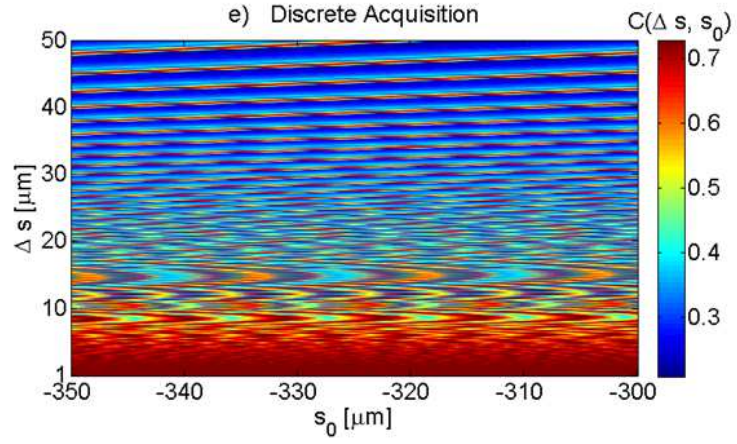

FIG. 2. EI signals of a PET wire (diameter $500 \mu \mathrm{m}$ ), simulated with step sizes $(\Delta s)$ of $50 \mu \mathrm{m}, 20$ $\mu \mathrm{m}$ and $1 \mu \mathrm{m}(\mathrm{a}-\mathrm{c})$. Contrast obtainable from continuous and discrete acquisition schemes as a function of step size $(\Delta s)$ and the position of the object at the start of the scan $\left(s_{0}\right)(\mathrm{d}, \mathrm{e})$.

For the continuous acquisition [Fig. 2(d)], this can be explained by the fact that the object movement effectively acts as a low pass filter on the ideal signal. In Eq. 8, this is represented by the convolution with a box function of width $\Delta s$. By assuming that to first approximation the sinc function (i.e. the Fourier transform of the box function) assumes negligible values beyond its first root, the cut-off frequency induced by the low pass filter is given by the inverse of the step size, i.e. $f_{c u t}=1 / \Delta s$. Hence, smaller step sizes correspond to higher cut-off frequences, which means that fringes are less smeared and contrast is preserved. The filtering also explains why the contrast in Fig. 2(d) is almost independent from $s_{0}$ : for lower sampling rates the fringes are blurred, hence they are less sensitive to 


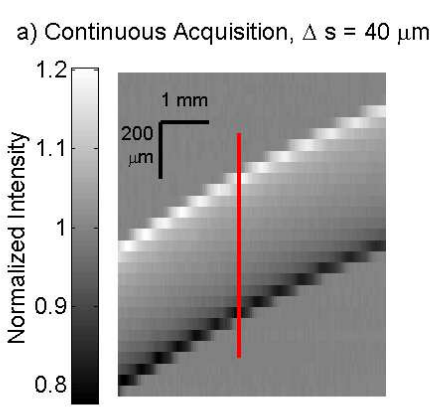

d) Continuous Acquisition, $\Delta \mathrm{s}=20 \mu \mathrm{m}$

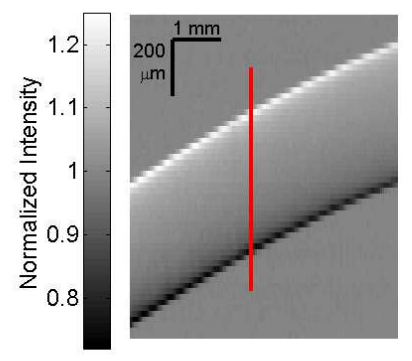

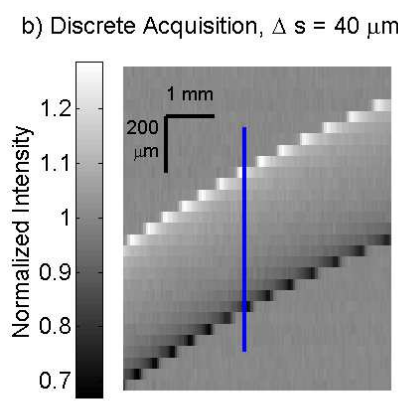

e) Discrete Acquisition, $\Delta \mathrm{s}=20 \mu \mathrm{m}$

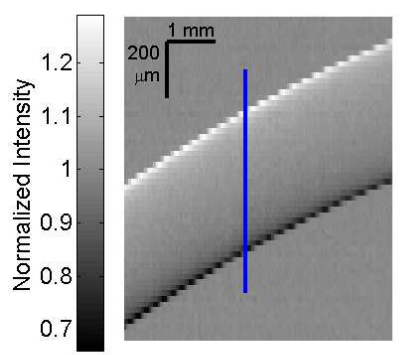

c) Profile Plots, $\Delta s=40 \mu \mathrm{m}$

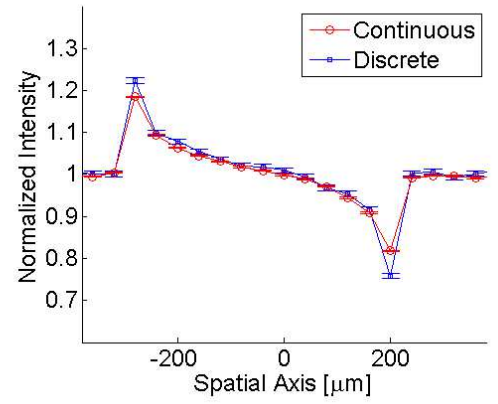

f) Profile Plots, $\Delta s=20 \mu \mathrm{m}$

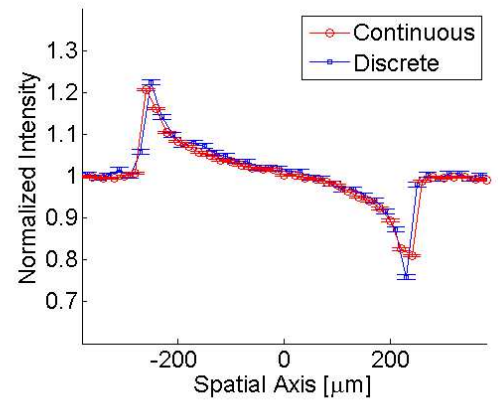

FIG. 3. Experimental images of a PET wire acquired with continuous and discrete acquisition schemes and step sizes of $\Delta s=40 \mu \mathrm{m}(\mathrm{a}, \mathrm{b})$ and $\Delta s=20 \mu \mathrm{m}(\mathrm{d}, \mathrm{e})$. Profiles extracted from the experimental images $(\mathrm{c}, \mathrm{f})$.

where they are sampled.

Conversely, for the discrete acquisition [Fig. 2(e)], contrast shows a strong dependence on $s_{0}$. This can be understood by noting that in this case no filtering occurs, and the ideal signal is sampled directly; thus, high spatial frequencies (which translate into sharper phase fringes) are preserved. Consequently, when large step sizes are employed, it is unlikely that the fringes are sampled exactly at the peaks where maximum contrast occurs, although this is in principle possible for a few values of $s_{0}$. As the sampling rate increases, this becomes more likely, hence the increased contrast and reduced dependence on $s_{0}$.

To some extent, one may consider the discrete scheme superior in terms of contrast since very high contrast values can potentially be obtained at low sampling rates, which is not possible with a continuous scheme. However, it should be noted that while in a simulation study it is possible to investigate the effect of $s_{0}$ and consequently select this parameter in such a way that contrast is maximized, $s_{0}$ effectively assumes random values in a real experiment. 
Figure 3 shows experimental data of a PET wire acquired with continuous and discrete acquisition schemes: the top and bottom rows show data obtained with step sizes of 40 $\mu \mathrm{m}$ and $20 \mu \mathrm{m}$, respectively. Besides the obvious fact that images obtained with a higher sampling rate $(\Delta s=20 \mu \mathrm{m})$ have better resolution, Fig. 3(a) clearly shows the blur induced by the continuous acquisition. Contrast in each images was calculated on a column by column basis according to Eq. 9 and averaged. For a step size of $40 \mu \mathrm{m}$, this yielded $0.16 \pm$ 0.02 and $0.19 \pm 0.05$ contrast for the continuous and the discrete acquisitions, respectively. For a step size of $20 \mu \mathrm{m}$, contrast was $0.21 \pm 0.02$ (continuous aquisition) and $0.23 \pm 0.03$ (discrete acquisition). The errors represent one standard deviation of all values averaged to obtain the results, and indicate the contrast variation across the images. The values are in agreement with the predictions of Figs. 2(d) and (e): for both acquisition schemes, contrast is increased for the higher sampling rate. Moreover, the contrast variation is smaller for the continuous than the discrete scheme, confirming the weaker dependence on the parameter $s_{0}$. Figs. 3(c) and (f) show profiles extracted along the indicated lines. The error bars represent the noise level in the background of the images.

\section{B. Effect on Phase Shift}

Figure 4 illustrates how continuous and discrete acquisition schemes, as well as the sampling rate, influence the phase shift that can be retrieved from two EI signals obtained under opposing EI conditions. Panels 4(a) to (c) show phase shift profiles that were retrieved from signals simulated for step sizes of 50,20 and $1 \mu \mathrm{m}$ (corresponding to sampling rates of 0.02, 0.05 and 1 samples $/ \mu \mathrm{m})$, respectively. It can be seen that already for $\Delta s=50 \mu \mathrm{m}$ the phase shift retrieved through a continuous scheme is a good approximation of the ideal case, apart from a slight blur at the edges and a small underestimation in the centre of the wire. In comparison, the profile obtained through a discrete scheme differs significantly from the ideal case: the phase shift is overestimated within the wire, and does not come back to the baseline at the end of the wire, leading to a gross underestimation outside it. The discrete signal improves for $\Delta s=20 \mu \mathrm{m}$, and for $\Delta s=1 \mu \mathrm{m}$ it becomes practically identical to both the continuous and ideal case.

Figs. $4(\mathrm{~d})$ and (e) show the maximum phase shift $\left(\Phi_{\max }\right)$ retrieved from all simulations $\left(\Delta s=50 \mu \mathrm{m}\right.$ to $0.1 \mu \mathrm{m}, s_{0}=-350 \mu \mathrm{m}$ to $\left.-300 \mu \mathrm{m}\right)$ as a function of step size $(\Delta s)$ and 
a) $\Delta \mathrm{s}=50 \mu \mathrm{m}, \mathrm{s}_{0}=-350 \mu \mathrm{m}$

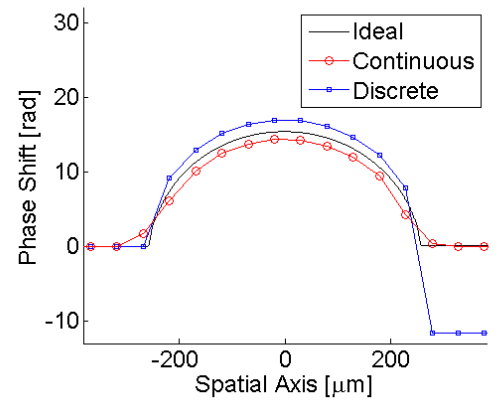

d) Continuous Acquisition

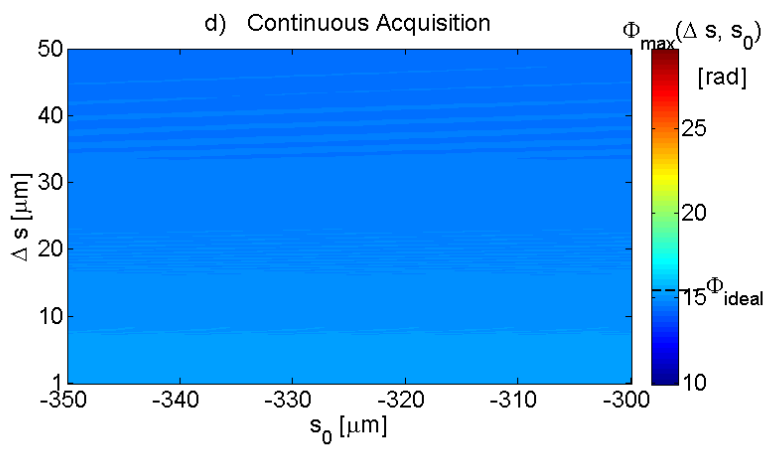

b) $\Delta \mathrm{s}=20 \mu \mathrm{m}, \mathrm{s}_{0}=-350 \mu \mathrm{m}$

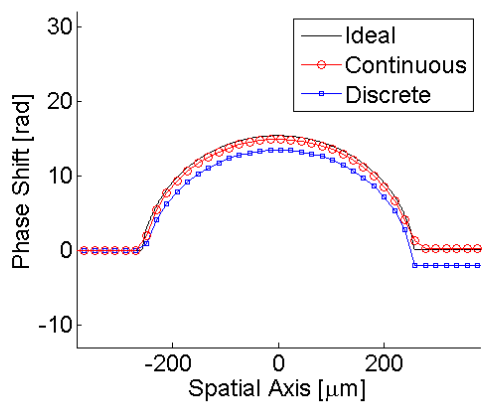

c) $\Delta \mathrm{s}=1 \mu \mathrm{m}, \mathrm{s}_{0}=-350 \mu \mathrm{m}$

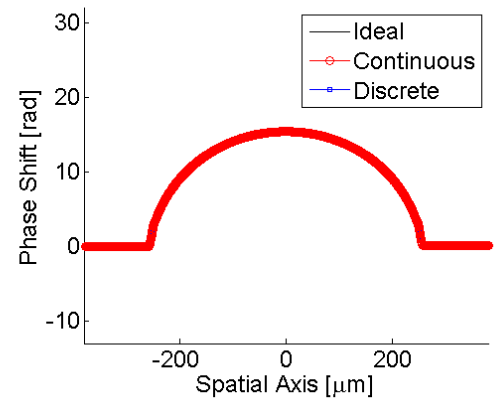

e) Discrete Acquisition

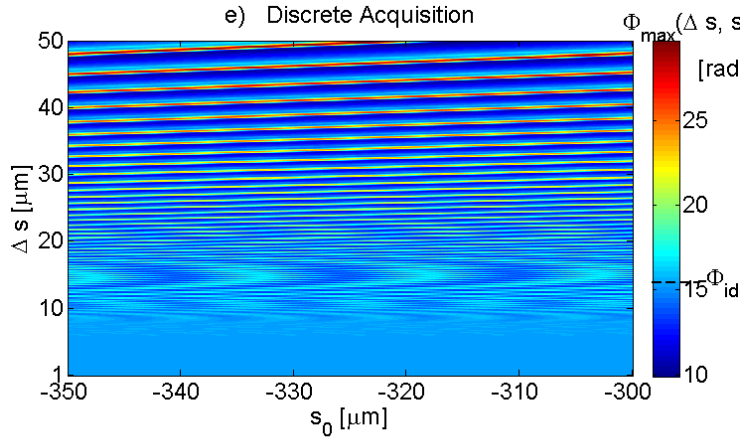

FIG. 4. Phase shift profiles extracted from two signals of a PET wire (diameter $500 \mu \mathrm{m}$ ) that were simulated under opposing edge illumination conditions with step sizes $(\Delta s)$ of $50 \mu \mathrm{m}, 20 \mu \mathrm{m}$ and $1 \mu \mathrm{m}(\mathrm{a}-\mathrm{c})$. Maximum retrieved phase shift $\left(\Phi_{\max }\right)$ from discrete and continuous acquisitions as a function of the step size $(\Delta s)$ and the position of the object at the start of the scan $\left(s_{0}\right)(\mathrm{d}, \mathrm{e})$.

position of the object at the start of the scan $\left(s_{0}\right)$. The phase shift value retrieved from the ideal signals $\left(\Phi_{\text {ideal }}=15.04 \mathrm{rad}\right)$ is indicated by the dashed lines in the colour bars in both panels. The general behaviour of the retrieved phase shift reflects what was discussed in relation to Figs. 4(a) to (c). In the plot for the continuous scheme [Fig. 4(d)], the phase shift shows a high accuracy and a weak dependence on $s_{0}$, which is a result of near optimal sampling: since the sampling rate is always equal to the cut-off frequency, it is smaller than the Nyquist frequency by only a factor of two $\left(f_{s}=f_{c u t}=0.5 f_{N}\right)$. Alternatively, the high accuracy can be understood by noting that, in the continuous case, the extracted refraction angle can be expressed via the following integral, in analogy to Eq. 7:

$$
\alpha\left(\Delta s, s_{0}, n\right) \approx \frac{1}{\Delta s} \int_{-\Delta s / 2}^{\Delta s / 2} \alpha_{\text {ideal }}\left(s_{0}+\left(n^{\prime}-1\right) \Delta s-s^{\prime}\right) d s^{\prime}
$$

i.e. in terms of refraction angle that would be extracted from ideal signals (ideal). If this is 
inserted into Eq. 10, the following relation can be obtained:

$$
\Phi\left(\Delta s, s_{0}, n\right) \approx \frac{2 \pi}{\lambda} \int_{s_{0}}^{s_{0}+n \Delta s} \alpha_{\text {ideal }}\left(s^{\prime}\right) d s^{\prime}=\Phi_{\text {ideal }}\left(s_{0}+n \Delta s\right) .
$$

Hence, in a continuous acquisition, the retrieved phase shift is effectively a sampled version of the phase shift obtained from ideal signals. For a fixed value of $\Delta s$, the maximum of the sampled phase shift can be slightly smaller than that of the ideal phase shift, depending on $s_{0}$. However, phase values have a much higher degree of accuracy compared to a standard discrete acquisition. For example, a step size of $50 \mu \mathrm{m}$ yields the phase shift with a maximum error of $11 \%$, and for step sizes smaller than $40 \mu \mathrm{m}$ the error is below $10 \%$.

In contrast, for the discrete scheme [Fig. 4(e)], the accuracy of the retrieved phase shift is strongly influenced by the parameter $s_{0}$, which in the worst case can lead underestimations and overestimations of up to $80 \%$ compared to the nominal value. This can be understood by noting that, if the individual EI signals are undersampled [Figs. 2(a) and (b)], so is the extracted refraction angle, which can cause problems during the retrieval of the phase shift. For example, if the left phase fringe is not sampled at its peak, the integration of the refraction angle can yield an underestimated phase shift. Conversely, if the fringe is sampled very close to the peak, Eq. 10 yields an overestimated phase shift due to the use of the sum rule: the multiplication of the peak value times a large step size leads to an excessive weight being assigned to it. For the same reasons, the phase shift can be under- or overestimated on the right hand side of the wire. When the sampling rate is increased, this effect becomes weaker as the phase fringes are more accurately sampled (which also means that the sum in Eq. 10 becomes a better representation of the integration operation). Hence, to reliably retrieve the phase shift from a discrete acquisition with a high accuracy, a high sampling rate is required ${ }^{17}$. For example, to achieve an accuracy of $10 \%$ independent of $s_{0}$, a step size below $9.5 \mu \mathrm{m}$ is necessary. Note also that this value matches the intrinsic resolution limit of the system (see Section 3); implying that the Nyquist rate, which in this case is $1 /(2 \times 9.5)$ samples $/ \mu \mathrm{m}$, is approximately met. As a consequence, for this combination of object and experimental parameters, the discrete scheme requires about four times more data than the continuous scheme in order to reliably retrieve the phase shift with the same accuracy.

It should be highlighted that the latter observation holds for planar EI scans only. As was shown previously, when the method is used as a CT modality, accurate quantitative information can also be retrieved with low sampling rates from a discrete scheme ${ }^{17}$. This 
can be understood intuitively: undersampling artefacts "average out" during the CT reconstruction process, since data is effectively measured multiple times (for each projection angle).

Figure 5 shows phase shift images obtained from experimental data acquired with continuous and discrete acquisition schemes: the top and bottom rows show data obtained with step sizes of $40 \mu \mathrm{m}$ and $20 \mu \mathrm{m}$, respectively. The maximum phase shifts were calculated on a column by column basis according to Eq. 11 and averaged. For a step size of $40 \mu \mathrm{m}$, this yielded phase shifts of $16.1 \pm 0.6 \mathrm{rad}$ and $16.2 \pm 2.6 \mathrm{rad}$ for the continuous and the discrete acquisition, respectively. For a step size of $20 \mu \mathrm{m}$, the maximum retrieved phase shifts were $16.1 \pm 0.6 \mathrm{rad}$ (continuous aquisition) and $16.2 \pm 0.9 \mathrm{rad}$ (discrete acquisition). The errors represent one standard deviation of all values averaged to obtain the results, and are an indication of the phase shift variation across the images. Note that due to the binning (see Section 4), the values for $\Delta s=40 \mu \mathrm{m}$ and $\Delta s=20 \mu \mathrm{m}$ are expected to be similar in the continuous case. The fact that errors are generally smaller for the continuous than for the discrete scheme confirms the predictions of Figs. 4(d) and (e), and indicate a more reliable retrieval of accurate phase shift values. The large errors in the discrete case are a result of the strong dependence of the retrieved phase shift on $s_{0}$, which effectively varies horizontally due to the inclination of the wire. This explains the stripy appearance of the discrete images, which is especially pronounced for the lower sampling rate [Fig. 5(b)]. Figs. 5(c) and (f) show profiles extracted along the indicated lines. The error bars represent the noise level in the background of the images. Profiles show the substantial superiority of the continuous acquisition scheme for low sampling rates, as the phase underestimation on the right hand side of the wire caused by the discrete scheme in this case is effectively eliminated.

\section{CONCLUSIONS}

We discussed an alternative acquisition scheme for EI imaging based on a continuous instead of discrete object movement, and evaluated its performance using the exemplary case of a wire phantom and realistic experimental parameters. Criteria for comparison between the new and the previously used acquisition schemes were achievable contrast and accuracy

of the retrieved phase shift, as a function of the sampling rate. Theoretical conclusions were supported by experimental results obtained at beamline ID17 of the ESRF. 
a) Continuous Acquisition, $\Delta \mathrm{s}=40 \mu \mathrm{m}$

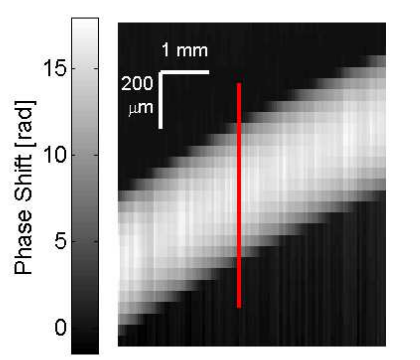

d) Continuous Acquisition, $\Delta s=20 \mu \mathrm{m}$

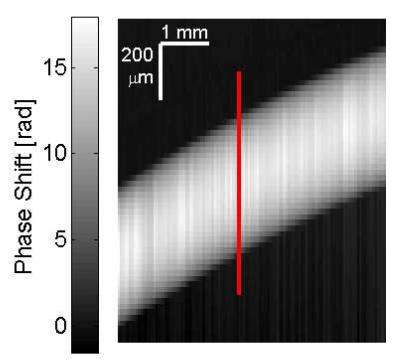

b) Discrete Acquisition, $\Delta \mathrm{s}=40 \mu \mathrm{m}$

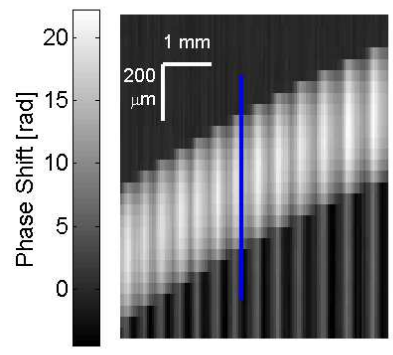

e) Discrete Acquisition, $\Delta s=20 \mu \mathrm{m}$

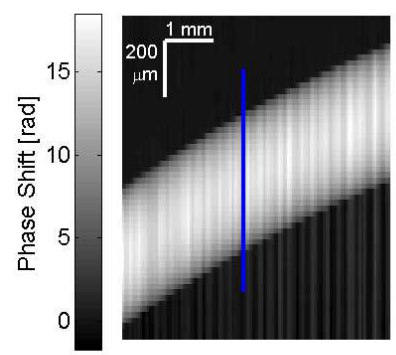

c) Profile Plots, $\Delta \mathrm{s}=40 \mu \mathrm{m}$

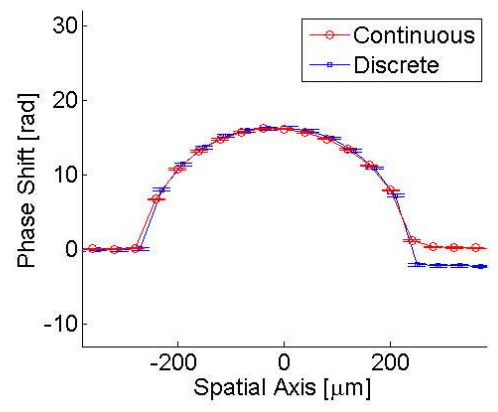

f) Profile Plots, $\Delta s=20 \mu \mathrm{m}$

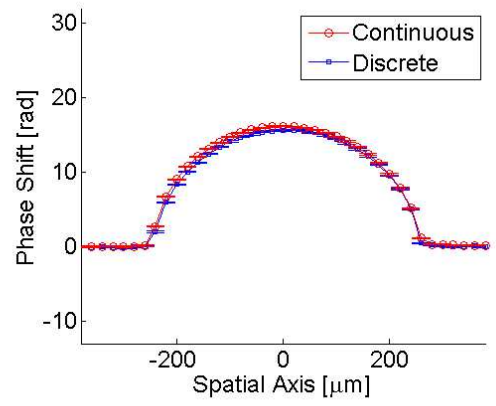

FIG. 5. Experimental images showing the phase shift of a PET wire retrieved from data acquired with discrete and continuous acquisition schemes and step sizes of $\Delta s=40 \mu \mathrm{m}(\mathrm{a}, \mathrm{b})$ and $\Delta s=20$ $\mu \mathrm{m}(\mathrm{d}, \mathrm{e})$. Profiles extracted from the experimental images $(\mathrm{c}, \mathrm{f})$.

The results indicate that contrast in unprocessed (i.e. non-phase retrieved) images is generally reduced by low sampling rates. For the continuous acquisition scheme, this is a result of the signal blur induced by the object movement. No blurring occurs during discrete scans, hence high frequencies are preserved, but contrast ultimately depends on the exact locations of the sampling points, determined by the position of the object at the start of the scan. The results further indicate that, when quantitatively accurate phase shift images are required, continuous acquisition schemes perform better than discrete ones. Although during a continuous scan a degree of blur is introduced, accurate phase shift values can be retrieved even for low sampling rates. In order to reliably achieve a comparable accuracy with a discrete scheme, significantly more data are required. An important consequence is that scans can be performed with less dose compared to discrete ones, which is a clear advantage for biological applications where low dose delivery is important. Moreover, continuous scans are generally faster than discrete ones.

On the other hand, discrete acquisitions could be advantageous in situations where quan- 
titative phase retrieval is not required, due to the reduced blurring typically leading to higher overall peak-to-peak contrast. For example, if the purpose is an improved visualization/detection of faint details, a single "mixed" (attenuation plus edge enhancement due to phase effects) image could be sufficient, and indeed this is the strategy adopted by e.g. the in vivo mammography program underway at the Trieste synchrotron ${ }^{23}$. It should be noted that, in the absence of additional sample scanning, this is effectively the case in the adaptation of the edge illumination method to conventional sources through masks featuring multiple apertures. A "single shot" (mixed) acquisition in that case effectively provides a discretely sampled image with sampling step equal to the pixel size, and indeed that approach was proven to yield significantly improved visibility in the imaging of beast tissue ${ }^{24}$ and other areas.

\section{ACKNOWLEDGMENTS}

This work was supported by the UK Engineering and Physical Sciences Research Council (Grant Nos. EP/L001381/1 and EP/I021884/1). PCD is supported by Marie Curie Career Integration Grant No. PCIG12-GA-2012-333990 within the Seventh Framework Programme of the European Union. Authors thank the ESRF (Grenoble, France) for having provided beamtime at their facilities and the ID17 staff for their support in image acquisition.

\section{REFERENCES}

${ }^{1}$ A. Bravin, P. Coan, and P. Suortti, "X-ray phase-contrast imaging: from pre-clinical applications towards clinics," Physics in Medicine and Biology 58(1), R1-R35 (2013)

${ }^{2}$ U. Bonse and M. Hart, "An x-ray interferometer," Applied Physics Letters 6(8), 155-156 (1965)

${ }^{3}$ V.N. Ingal and A. Beliaevskaya, "X-ray phase-wave topography observation of the phase contrast from a non-crystalline object," Journal of Physics D: Applied Physics 28(11), 2314-2317 (1995).

${ }^{4}$ T. Davis, D. Gao, T.E. Gureyev, A.W. Stevenson, and S.W. Wilkins, "Phase-contrast imaging of weakly absorbing materials using hard x-rays," Nature 373(6515), 595-598 (1995). 
${ }^{5}$ A. Snigirev, I. Snigireva, V. Krohn, S. Kuznetsov, and I. Schelokov, "On the possibilities of x-ray phase contrast microimaging by coherent high energy synchrotron radiation," Review of Scientific Intruments 66, 5486-5492 (1995).

${ }^{6}$ A. Olivo, F. Arfelli, G. Cantatore, R. Longo, R. Menk, S. Pani, M. Prest, P. Poporat, L. Rigon, G. Tromba, E. Vallazza, and E. Castelli, "An innovative digital imaging set-up allowing a low-dose approach to phase contrast applications in the medical field," Medical Physics 28(8), 1610-1619 (2001).

${ }^{7}$ A. Momose, S. Kawamoto, I. Koyama, Y. Hamaishi, K. Takai, and Y. Suzuki, "Demonstration of x-ray Talbot interferometry," Japanese Journal of Applied Physics 42(Part2, No. 7B), L866-L868 (2003).

${ }^{8}$ P.C. Diemoz, M. Endrizzi, C.E. Zapata, Z. Pesic, C. Rau, A. Bravin, I. Robinson, and A. Olivo, "X-ray phase contrast imaging with nanoradian angular resolution," Physical Review Letters 110, 138105 (2013).

${ }^{9}$ M. Endrizzi, F.A. Vittoria, P.C. Diemoz, R. Lorenzo, R.D. Speller, U.H. Wagner, C. Rau, I.K. Robinson, and A. Olivo, "Phase-contrast microscopy at high x-ray energy with a laboratory setup," Optics Letters 39(11), 3332-3335 (2014)

${ }^{10}$ C.K. Hagen, P.C. Diemoz, M. Endrizzi, L. Rigon, D. Droessi, F. Arfelli, F.C.M. Lopez, R. Longo, and A. Olivo, "Theory and preliminary experimental verification of quantitative edge illumination x-ray phase contrast tomography," Optics Express 22(7), 7989-8000 (2014).

${ }^{11} \mathrm{~A}$. Olivo and R. Speller, "A coded-aperture technique allowing x-ray phase contrast imaging with conventional sources," Applied Physics Letters 91, 074106 (2007).

${ }^{12}$ M. Endrizzi, F.A. Vittoria, G. Kallon, D. Basta, P.C. Diemoz, A. Vincenzi, P. Delogu, R. Bellazzini, and A. Olivo, "Achromatic approach to phase-based multi-modal imaging with conventional sources," Optics Express 23(12), 16473-16480 (2015).

${ }^{13}$ A. Olivo and R. Speller, "Image formation principles in coded-aperture based x-ray phase contrast imaging," Physics in Medicine and Biology 53(22), 6461-6474 (2008)

${ }^{14}$ P.R.T. Munro, C.K. Hagen, M.B. Szafraniec, and A. Olivo, "A simplified approach to quantitative coded aperture x-ray phase imaging," Optics Express 21(9), 11187-11201 (2013).

${ }^{15}$ C.K. Hagen, P.R.T. Munro, M. Endrizzi, P.C. Diemoz, and A. Olivo, "Low-dose phase contrast tomography with conventional sources," Medical Physics 41(7) 070701 (2014). 
${ }^{16}$ P.C. Diemoz, F.A. Vittoria, and A. Olivo, "Spatial resolution of edge illumination x-ray phase contrast imaging," Optics Express 22(13), 15514-15529 (2014).

${ }^{17}$ C.K. Hagen, P.C. Diemoz, M. Endrizzi, and A. Olivo, "The effect of the spatial sampling rate on quantitative phase information extracted from planar and tomographic edge illumination x-ray phase contrast images," Journal of Physics D: Applied Physics 47(45), $455401(2014)$

${ }^{18}$ H. Nyquist, "Certain topics in telegraph transmission theory," Trans. AIEE 47, 617-644 (1928).

${ }^{19}$ C.E. Shannon, "Communications in the presence of noise," Proc. Institute of Radio Engineers 37(1), 10-21 (1949).

${ }^{20}$ F.A. Vittoria, P.C. Diemoz, M. Endrizzi, L. Rigon, F.C. Lopez, D. Dreossi, P.R.T. Munro, and A. Olivo, "Strategies for efficient and fast wave optics simulation of coded-aperture and other x-ray phase contrast imaging methods," Applied Optics 52(28), 6940-6947 (2013).

${ }^{21}$ M.J. Berger, J.H. Hubbell, S.M. Seltzer, J. Chang, J.S. Coursey, R. Sukumar, D.S. Zucker, and K. Olsen, "XCOM: Photon Cross Section Database (version 1.5)," Online Available: http://physics.nist.gov/xcom [Wednesday, 22-Apr-2015 07:43:46 EDT], National Institute of Standards and Technology, Gaithersburg, MD (2010)

${ }^{22}$ P. Coan, A. Peterzol, S. Fiedler, C. Punchut, L.C. Labiche, and A. Bravin, "Evaluation of imaging performance of a taper optics CCD "FReLoN" camera designed for medical imaging," Journal of Synchrotron Radiation 13(Pt. 3), 260-270 (2006)

${ }^{23}$ E. Castelli, M. Tonutti, F. Arfelli, R. Longo, E. Quaia, D. Sanabor, F. Zanconati, D. Dreossi, A. Abrami, A. Quai, P. Bregant, K. Casarin, V. Chenda, R.H. Menk, T. Rokvoc, A. Vascotto, G. Tromba, and M.A. Cova, "Mammography with synchrotron radiation: first clinical experience with phase-detection technique," Radiology 259(3), 684-694 (2011)

${ }^{24}$ A. Olivo, S. Gkoumas, M. Endrizzi, C.K. Hagen, M.B. Szafraniec, P.C. Diemoz, P.R.T. Munro, K. Ignatyev, B. Johnson, J.A. Horrocks, S.J. Vinnicombe, J.L. Jones, and R.D. Speller, "Low-dose phase contrast mammography with conventional sources," Medical Physics 40(9), 090701 (2013). 